\title{
Volume Effect on the Magnetism of $\mathrm{Fe}_{3-x} \mathrm{Cr}_{x} \mathrm{Al}$
}

\author{
A. $\mathrm{GO}^{a}$, M. Pugaczowa-Michalska ${ }^{b}$ And L. Dobrzyński ${ }^{a, c}$ \\ ${ }^{a}$ Faculty of Physics, University of Białystok \\ Lipowa 41, 15-424 Białystok, Poland \\ ${ }^{b}$ Institute of Molecular Physics, Polish Academy of Sciences \\ M. Smoluchowskiego 17, 60-179 Poznań, Poland \\ ${ }^{c}$ The Sołtan Institute for Nuclear Studies, 05-400 Otwock-Świerk, Poland
}

\begin{abstract}
Magnetic properties of $\mathrm{Fe}_{3-x} \mathrm{Cr}_{x} \mathrm{Al}$ alloys depend strongly on the unit cell volume. Purpose of this work is to investigate the dependence of the total and local magnetic moments on the lattice constant using the self-consistent spin-polarised TB-LMTO method.
\end{abstract}

PACS numbers: 71.20.Be

\section{Introduction}

$\mathrm{Fe}_{3-x} \mathrm{Cr}_{x} \mathrm{Al}$ alloy, in the range of $0 \leq x \leq 0.5$, crystallises in the $\mathrm{DO}_{3}$-type structure. There are two non-equivalent $\mathrm{Fe}$ sites in the perfectly ordered $\mathrm{Fe}_{3} \mathrm{Al}$ structure. One of them, B-site, has eight Fe nearest neighbours in an octahedral configuration, and the latter, $(\mathrm{A}, \mathrm{C})$-site, has four $\mathrm{Fe}$ and four $\mathrm{Al}$ nearest neighbours. As a consequence of different environments, there are different electronic and magnetic properties connected with these iron positions. Especially, a decrease in the magnetic moment of iron with an increase in the chromium concentration was obtained by the Mössbauer measurements [1]. Our ab initio total energy calculations of $\mathrm{Fe}_{3-x} \mathrm{Cr}_{x} \mathrm{Al}$ strongly suggest that $\mathrm{Cr}$ atoms prefer to occupy B sublattice [2] which is in full agreement with the experimental results $[1,3,4]$. However, detailed theoretical investigations $[2,5]$ for the experimental values of a lattice constant [1] show a little bit different gradient of the total magnetic moment versus a lattice constant. Furthermore, there is quite unexpected behaviour of the magnetic moment of iron atoms on one of the unequivalent positions (B) for which the magnetic moment increases with the concentration of chromium.

It is well known that magnetic moment of pure iron depends strongly on a value of the Wigner-Seitz radius [6]. Thus we suspect that also in $\mathrm{Fe}_{3-x} \mathrm{Cr}_{x} \mathrm{Al}$ alloys volume effect is of great importance while considering magnetic properties. In this contribution we concentrate mainly on the discussion of an influence of the lattice constant on magnetic moments in compound where iron is substituted by chromium.

\section{Method of calculations}

The electronic and the magnetic properties for $\mathrm{Fe}_{3-x} \mathrm{Cr}_{x} \mathrm{Al}$ alloys have been obtained within the framework of the local spin density approximation, using the self-consistent spin-polarised tight binding linear muffin-tin orbital in the atomic sphere approximation (TB-LMTO-ASA) method [7]. Calculations were carried out for the exchange correlation potential in the form of von Barth and Hedin [8] for at least $280 k$-points in the irreducible wedge of the Brillouin zone. The LangrethMehl-Hu non-local exchange-correlation correction [9] was added. Iterations were repeated until the energy eigenvalues of the consecutive iteration steps were the same within the range of $0.01 \mathrm{mRy}$. Supercell structure of 32 atoms was used in calculations. For one chromium concentration there were carried out total energy calculations for the experimental lattice constant in order to find such an atomic configuration which minimises the total energy [2]. Next for this atomic configuration we have investigated an influence of a changing lattice constant on the magnetic properties. The band structure and the magnetic moments were computed for the changing values of the lattice constant in the range of $\pm 10 \%$ in comparison with experimental values [1]. It was assumed that $\mathrm{Al}$ atoms occupy $\mathrm{D}$ positions, $\mathrm{Cr}$ locates at $\mathrm{B}$ sites only (according to the experimental results $[1,3,4]$ and our previous investigations [2]), whereas Fe atoms occupy both $(\mathrm{A}, \mathrm{C})$ and $\mathrm{B}$ positions. Calculations were performed for five values of the concentration parameter $x: 0,0.125$, $0.25,0.375$ and 0.5 .

\section{Results}

An increase in the local and total magnetic moments versus an increase in the lattice parameter is observed.

For the experimental lattice constant, which slightly decreases with the increasing chromium content, the total magnetic moment of $\mathrm{Fe}_{3} \mathrm{Al}$ obtained experimentally [1] is slightly lower than the calculated one (Fig. 1). We obtained the same, as measured, total magnetic moment's value for about $5 \%$ smaller lattice constant. When concentration of $\mathrm{Cr}$ increases, difference between the exper- 


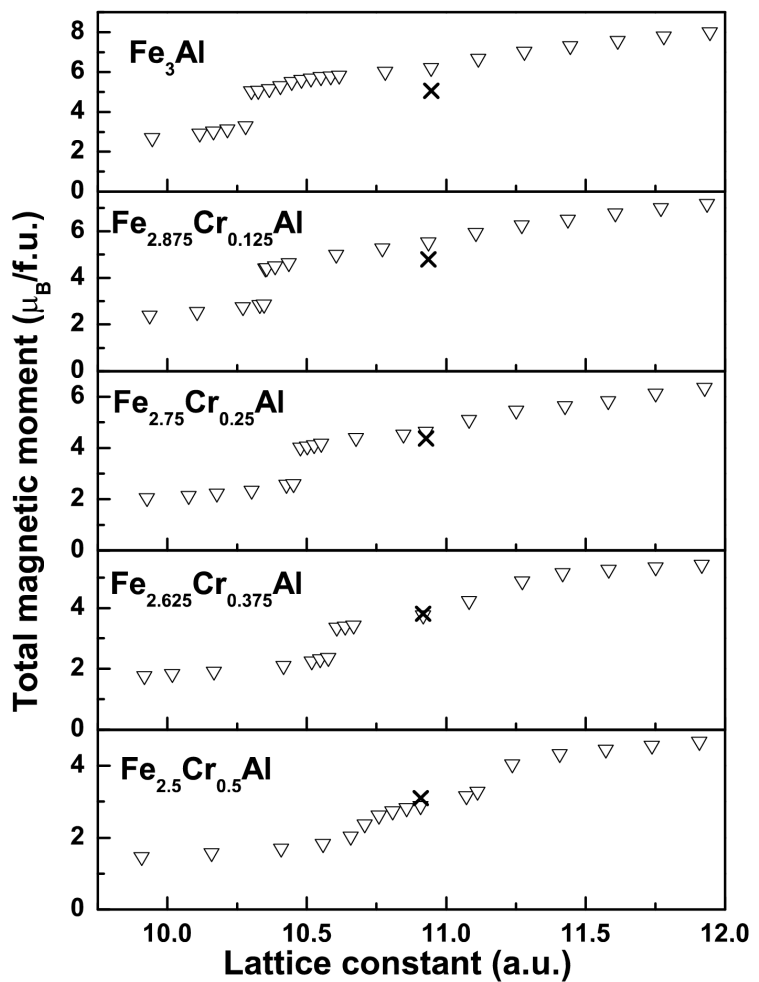

Fig. 1. Dependence of the $\mathrm{Fe}_{3-x} \mathrm{Cr}_{x} \mathrm{Al}$ total magnetic moment on lattice constant. Cross symbols indicate experimental values [1].

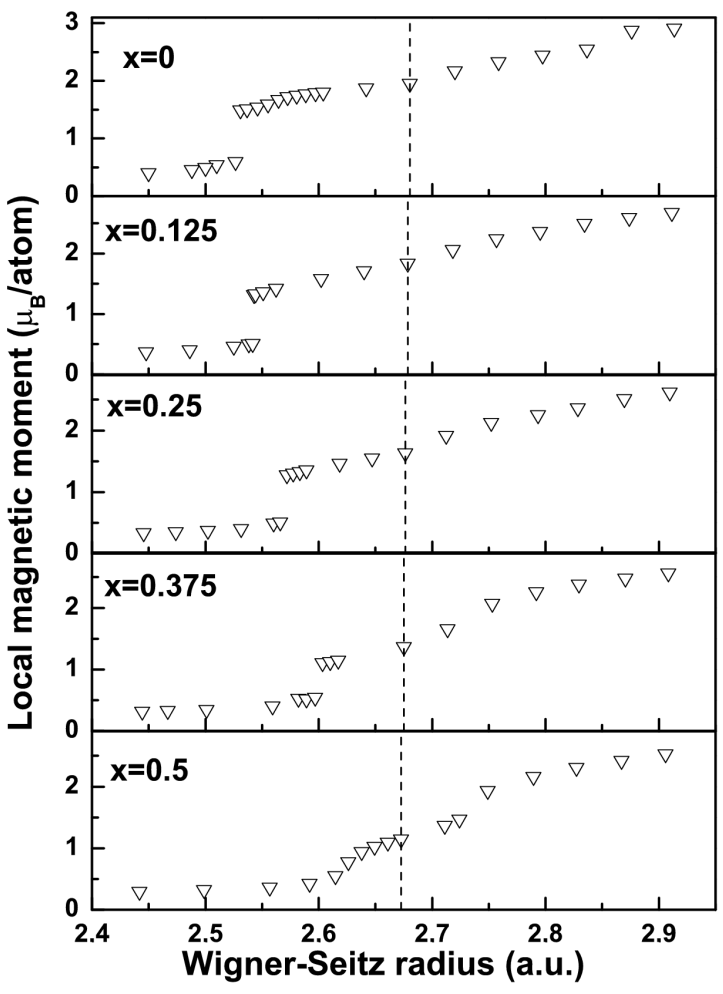

Fig. 2. Dependence of the Fe(A,C) magnetic moment on a Wigner-Seitz radius. The dashed lines mark the Wigner-Seitz radii corresponding to the experimental lattice constants.

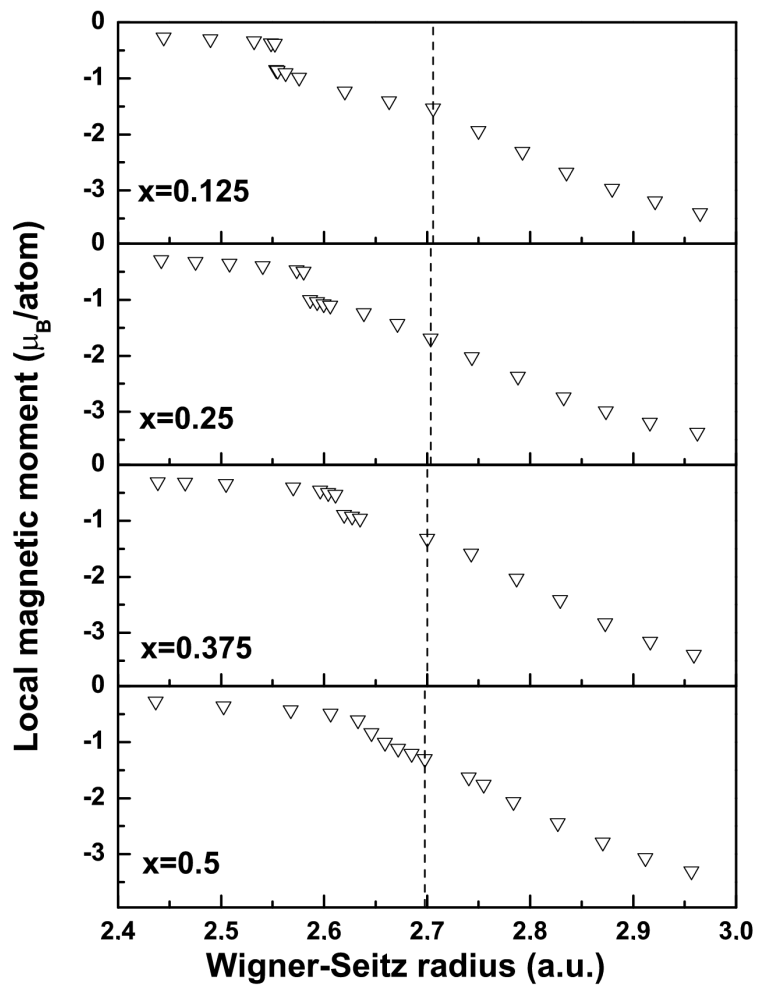

Fig. 3. Dependence of the $\mathrm{Cr}(\mathrm{B})$ magnetic moment on a Wigner-Seitz radius. The dashed lines mark the Wigner-Seitz radii corresponding to the experimental lattice constants.

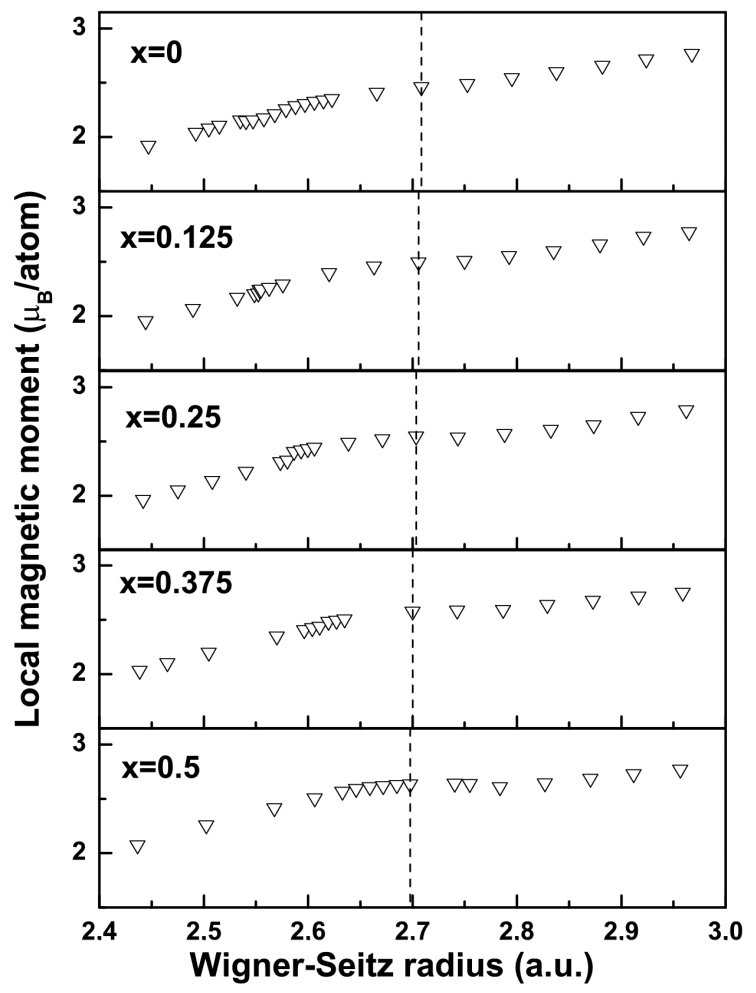

Fig. 4. Dependence of the $\mathrm{Fe}(\mathrm{B})$ magnetic moment on a Wigner-Seitz radius. The dashed lines mark the Wigner-Seitz radii corresponding to the experimental lattice constants. 
imental value of the total magnetic moment and a theoretical value calculated for experimental lattice constant decreases. For $x=0.375$ these values are equal within the error range. In order to obtain the total magnetic moment such as measured for $x=0.5$, the value of lattice parameter was assumed higher than experimental one by about $2 \%$.

Furthermore, when volume of the unit cell decreases compared to the measured volume, there is observed rapid drop of the total magnetic moment. In pure $\mathrm{Fe}_{3} \mathrm{Al}$ lattice constant corresponding to the abovementioned effect is about $6 \%$ smaller than experimentally obtained [1] and leads to above $30 \%$ reduction of the magnetic moment. For the non-zero concentrations of $\mathrm{Cr}$, value of the critical Wigner-Seitz radius slightly increases and the curve becomes smoother. This abrupt change of the total magnetic moment is connected mainly with changes of local magnetic moments of $\mathrm{Fe}(\mathrm{A}, \mathrm{C})$ (Fig. 2) as well as $\mathrm{Cr}(\mathrm{B})$ (Fig. 3) for doped alloys. If $x=0$, almost a $60 \%$ decrease in the $\mathrm{Fe}(\mathrm{A}, \mathrm{C})$ magnetic moment was obtained for the critical lattice constant. Small $\mathrm{Cr}$ content does not change a percentage drop of the $\mathrm{Fe}(\mathrm{A}, \mathrm{C})$ magnetic moment. Only when $x=0.375$ this drop decreases to $50 \%$. The similar rapid change of the magnetic moment was observed for pure fcc iron for the radius of the Wigner-Seitz sphere equal to about 2.7 a.u. [6]. That reduction of the magnetic moment is connected mainly with changes of the density of majority states $\mathrm{Fe}(\mathrm{A}, \mathrm{C})$ near the Fermi energy. For smaller $\mathrm{Cr}$ concentration $(x<0.375)$, a sharp big peak of majority density of states (DOS) is located below the Fermi level. A decrease in the lattice parameter causes a shift of this maximum towards the Fermi surface. Reaching of the critical value of the lattice constant leads to an abrupt change of $\mathrm{Fe}(\mathrm{A}, \mathrm{C})$ magnetic moment. For $x=0.5$, aforementioned peak is considerably smaller and wider and ipso facto less sensitive to changes of the Wigner-Seitz radius. In the minority $\mathrm{Fe}(\mathrm{A}, \mathrm{C})$ subband, $e_{\mathrm{g}}$ states are located partially below and above the Fermi energy and do not give essential contribution to the rapid drop of the magnetic moment.

Magnetic moment of $\mathrm{Cr}$ is antiparallel compared to $\mathrm{Fe}$ magnetic moments (Fig. 3). When volume of the unit cell decreases, a rapid drop of the absolute value of the chromium magnetic moment, for the same critical lattice parameter as for $\mathrm{Fe}(\mathrm{A}, \mathrm{C})$, is observed too. That drop is connected with the minority $\mathrm{Cr}$ states near the Fermi level.

Magnetic moments of iron atoms on B positions decrease more slightly in the considered range of lattice parameter (Fig. 4), differently to all other atoms. It is connected with $\mathrm{Fe}(\mathrm{B})$ electronic structure. Both majority and minority main peaks are located deeper below Fermi level and in the range of considered lattice parameter are not shifted, even partially, above Fermi energy.
The nearest neighbourhood of $\mathrm{Fe}(\mathrm{B})$ is the same like for pure $\mathrm{Fe}$ in bcc structure. Our result for $\mathrm{Fe}(\mathrm{B})$ is consistent with calculations for the bcc Fe [6].

A small negative magnetic moment induced on aluminium atoms depends also on the Wigner-Seitz radii. For the critical value of the lattice constant some drop of the magnetic moment occurs.

\section{Conclusions}

Results of the present study show that magnetic properties depend strongly on the unit cell volume. In pure $\mathrm{Fe}_{3} \mathrm{Al}$, the measured total magnetic moment [1], can theoretically be explained if the lattice constant was lower by about $5 \%$ from the experimental one [1]. This effect decreases with increasing chromium content. If the magnetic moments had been dependent on the lattice parameter only, one could expect that lattice parameter should rather increase with the chromium concentration (as in $\mathrm{Fe}_{3-x} \mathrm{Cr}_{x} \mathrm{Si}$ ) than decrease as observed in experiment [1]. In order to determine lattice parameter it is needed to carry out total energy calculations and possibly vary Wigner-Seitz radii. Nevertheless, the differences between experimental and theoretical findings indicate that local deformation of the lattice may play important role in observed features of $\mathrm{Fe}_{3-x} \mathrm{Cr}_{x} \mathrm{Al}$. The system behaves as if the lattice constant of the $\mathrm{Fe}_{3} \mathrm{Al}$ matrix was strained and exhibited larger lattice parameter than should. In such a case, chromium would release the strain.

\section{References}

[1] D. Satuła, L. Dobrzyński, J. Waliszewski, K. Szymański, K. Rećko, A. Malinowski, Th. Brückel, O. Schärpf, K. Blinowski, J. Magn. Magn. Mater. 169, 240 (1997).

[2] A. Go, M. Pugaczowa-Michalska, L. Dobrzyński, Phys. Status Solidi B 243, 1241 (2006).

[3] D. Satuła, K. Szymański, L. Dobrzyński, J. Waliszewski, A. Malinowski, J. Magn. Magn. Mater. 140144, 61 (1995).

[4] D. Satuła, L. Dobrzyński, A. Malinowski, K. Szymański, J. Waliszewski, J. Magn. Magn. Mater. 151, 211 (1995).

[5] A. Go, M. Pugaczowa-Michalska, L. Dobrzyński, Mol. Phys. Rep. 38, 86 (2003).

[6] D. Bagayoko, J. Callaway, Phys. Rev. B 28, 5419 (1983).

[7] O.K. Andersen, O. Jepsen, M. Sob, in: Electronic Structure and Its Applications, Ed. M. Yussouff, Lectures Notes in Physics, Vol. 283, Springer, Berlin 1987 , p. 1.

[8] U. von Barth, L. Hedin, J. Phys. C 5, 1629 (1972).

[9] C.D. Hu, D.C. Langreth, Phys. Scr. 32, 391 (1985). 